\title{
White Matter Disease and Outcomes of Mechanical Thrombectomy for Acute Ischemic Stroke
}

(D)E.A. Mistry, (D) A.M. Mistry, (D)T. Mehta, (D) N. Arora, (D) A.K. Starosciak, (D)F.D.L.R. La Rosa, (D).E. Siegler, (D) S.E. Kasner, (D) R. Chitale, (DM. Fusco, (DM. Froehler, (DS. Yaghi, DM. Schrag, and (DP. Khatri, on behalf of BEST Collaborators

\section{ABSTRACT}

BACKGROUND AND PURPOSE: The increased severity of white matter disease is associated with worse outcomes and an increased rate of intracerebral hemorrhage in patients with ischemic stroke undergoing thrombolytic treatment. However, whether white matter disease is associated with outcomes in patients undergoing endovascular treatment remains unclear.

MATERIALS AND METHODS: In this prespecified exploratory analysis of our prospective multi-institutional study that enrolled consecutive adult patients with anterior circulation ischemic stroke undergoing endovascular treatment from November 2017 to September 2018, we compared the following outcomes between patients with none-to-minimal (van Swieten score, 0-2) and moderate-to-severe (van Swieten score, 3-4) white matter disease using logistic regression: 90-day mRS 3-6, death, intracerebral hemorrhage, successful recanalization, and early neurologic recovery.

RESULTS: Of the 485 patients enrolled in the Blood Pressure after Endovascular Stroke Therapy (BEST) study, 389 had white matter disease graded (50\% women; median age, 68 years; range, 58-79 years). A van Swieten score of 3-4 $(n=74 / 389,19 \%)$ was associated with a higher rate of 90 -day mRS of $3-6$ (45\% versus $18 \%$; adjusted $\mathrm{OR}, 2.73 ; 95 \% \mathrm{Cl}, 1.34-5.93 ; P=.008)$. Although the death rate was higher in patients with van Swieten scores of 3-4 (26\% versus 15\%), the adjusted likelihood was not significantly different (adjusted OR, 1.14; 95\% Cl, 0.56-2.26; $P=.710$ ). Ordered regression revealed a shift toward worse mRS scores with increasing van Swieten scores (adjusted common OR, 3.04; 95\% Cl, 1.93-4.84; $P<.001$ ). No associations between white matter disease severity and intracerebral hemorrhage, successful recanalization, and early neurologic recovery were observed.

CONCLUSIONS: Moderate-to-severe white matter disease is associated with worse outcomes in patients undergoing endovascular treatment without a significant increase in hemorrhagic complications. Studies comparing patients with and without endovascular treatment are necessary to determine whether the benefit of endovascular treatment is attenuated with greater white matter disease.

ABBREVIATIONS: $\mathrm{aOR}=$ adjusted OR; EVT = endovascular treatment; ICH = intracerebral hemorrhage; $V$ SS = van Swieten score; WMD = white matter disease

E ndovascular treatment (EVT) drastically improves outcomes for select patients with an acute large-vessel occlusion and is the treatment technique with the highest level of evidence

Received December 10, 2019; accepted after revision January 31, 2020.

From the Departments of Neurology (E.A.M., M.S.) and Neurosurgery (A.M.M., R.C., M. Fusco), and Cerebrovascular Program (M. Froehler), Vanderbilt University Medical Center, Nashville, Tennessee; Department of Neurology and Neurosurgery (T.M.), University of Minnesota, Minneapolis, Minnesota; Department of Neurology (N.A.), University of Missouri, Columbia, Missouri; Baptist Health Neuroscience Center (A.K.S., F.D.L.R.L.R.), Miami, Florida; Department of Neurology (J.E.S., S.E.K.), University of Pennsylvania, Philadelphia, Pennsylvania; New York University Langone Health (S.Y.), Brooklyn, New York; and Department of Neurology and Rehabilitation Medicine (P.K.), University of Cincinnati, Cincinnati, Ohio.

This work was supported by the National Institutes of Health/National Institute of Neurological Disorders and Stroke (K23NS113858; Mistry), University of Cincinnati Gardner Neuroscience Institute pilot grant, and Society of Vascular and Interventional Neurology pilot grant. according to the most recent guidelines. ${ }^{1,2}$ Thus, the use of EVT as the definitive treatment technique for large-vessel occlusion strokes is increasing. ${ }^{3}$ Moreover, the indications for EVT are expanding to populations that were previously thought to not derive many benefits from EVT, such as those with delayed presentation. ${ }^{4,5}$ Additional populations that may derive benefit are also under study, such as those with a large infarct core, ${ }^{6,7}$ older age, ${ }^{8}$ and low NIHSS scores. ${ }^{9}$ White matter disease (WMD) is easily detectable on baseline routine imaging performed in all

Please address correspondence to Eva A. Mistry, MBBS, 2525 West End Ave, Suite 612, Nashville TN 37232; e-mail: eva.a.mistry@vumc.org

\footnotetext{
-- Indicates open access to non-subscribers at www.ajnr.org

$\equiv$ Indicates article with supplemental on-line tables.

http://dx.doi.org/10.3174/ajnr.A6478
} 
patients with stroke. Moderate-to-severe WMD is associated with poorer outcomes in patients with stroke from any etiology and increases the rate of hemorrhagic complications in those treated with intravenous thrombolysis. ${ }^{10,11}$ However, outcomes of patients with moderate-to-severe WMD in the context of EVT remain less well-studied, and the rates of early neurologic recovery and hemorrhagic complications remain unknown. In this prespecified exploratory analysis of our prospective, multicenter, cohort study, Blood Pressure after Endovascular Stroke Therapy (BEST), we sought to determine whether patients with acute stroke with moderate-to-severe WMD undergoing EVT have worse functional outcomes and increased rates of hemorrhagic complications compared with those with no-to-minimal WMD.

\section{MATERIALS AND METHODS \\ Study Design and Patient Selection}

The BEST study prospectively enrolled consecutive adult patients undergoing EVT for an anterior cerebral circulation large-vessel occlusion (internal carotid artery or the M1 or M2 segments of the middle cerebral artery) at 12 comprehensive stroke centers across the United States from November 2017 to September 2018. We excluded from the study patients with the following conditions: 1) a known prestroke mRS score of less than 2;2) terminal medical diagnoses such as a stage IV cancer; 3 ) a left ventricular assist device; or 4) a stroke in the perioperative or inpatient setting. Additional details on methods have been previously published. ${ }^{12}$ The BEST study was approved by the institutional review boards of 11 sites and was deemed exempt by 1 site, and informed consent was waived. The results presented here are a prespecified secondary analysis of the BEST study. The data that support the findings of this study are available from the corresponding author on reasonable request.

\section{Study Variables and Covariates}

The primary study variable was the severity of WMD, graded prospectively. A single physician collected data on WMD on the first CT scan at presentation for each patient using the van Swieten score (VSS), after undergoing centralized training on the grading. ${ }^{13}$ The VSS was graded on the contralateral hemisphere of the known large-vessel occlusion. This technique has been used by previous studies to avoid mislabeling the ipsilateral acute stroke-related hypodensities on the CT scan as WMD. ${ }^{14}$ Furthermore, the van Swieten method of WMD grading has been deemed comparable with other CT- and MR imaging-based grading systems. ${ }^{15}$

We collected the following variables: the ASPECTS, available from the radiology report of the first noncontrast brain CT or calculated by trained local study personnel; core and penumbra volumes automatically calculated by the RApid processing of PerfusIon and Diffusion software (RAPID; iSchemaView, Stanford, California) from the initial pre-EVT CT perfusion scan; time from last known well to final recanalization assessed as a modified TICI score (or angiogram completion if the final modified TICI was 0); and the presence of any intraparenchymal hemorrhage (ICH; excluding subarachnoid hemorrhage) on either MR imaging or CT within the 72 hours post-EVT.

\section{Outcomes}

The primary study outcome was the dichotomized 90-day mRS 0-2 (functional independence) versus 3-6. Secondary outcomes included a 90-day mRS 0-3 (ambulatory independence) versus 4-6, any ICH, symptomatic ICH (associated with a $\geq 4$-point increase in the NIHSS score from baseline to 24 hours), a change in the mRS distribution, successful recanalization (defined as modified TICI $2 b-3$ ), and early neurologic recovery (NIHSS 0-1 or $\geq 8$-point decrease in the NIHSS score from baseline to 24 hours).

\section{Statistical Analysis}

The baseline characteristics between the group of patients with no-to-minimal (VSS 0-2) and moderate-to-severe (VSS 3-4) WMD were compared using a $\chi^{2}$ test, $t$ test, or Mann-Whitney $U$ test, as appropriate. Associations between WMD with outcomes were determined using logistic regression. These models were subsequently adjusted for the following covariables selected a priori: age, hypertension, initial glucose level, NIHSS score on presentation, time to recanalization, intravenous thrombolysis, ASPECTS, systolic blood pressure on admission, and successful recanalization (except when used as the dependent variable). A subgroup analysis was then undertaken on only those patients with a successful recanalization. All analyses and plots were generated using R statistical and computing software, Version 3.6 (http://www.r-project.org/). Statistical significance $\alpha$ was set at $<.05$ for all statistical analyses. All $P$ values are 2 -sided.

\section{RESULTS}

Of the 485 patients included in BEST, 389 had WMD graded on the baseline CT scan, of whom 90-day follow-up was available for 365 (94\%). A total of 74 (19\%) had moderate-to-severe WMD (VSS 3-4). Table 1 outlines the baseline characteristics and outcomes according to moderate-to-severe and no-to-minimal WMD. Patients with VSS 3-4 were older and had a higher proportion with hypertension in addition to having a higher baseline blood glucose level and systolic blood pressure. The remaining baseline characteristics were not different between the 2 groups.

A total of 13 (18\%) of the 70 patients with VSS 3-4 had a 90day mRS 0-2 compared with 141 (45\%) of the 295 with VSS 0-2 $(P<.001$, Table 1$)$. Compared with patients with VSS $0-2$, those with VSS 3-4 had higher odds of being functionally dependent or dead (OR, 4.01; 95\% CI, 2.17-7.94; $P<.001$; adjusted OR [aOR], 2.73; 95\% CI, 1.34-5.93; $P=.008$ for having a 90-day mRS 3-6). The complete results of the model are depicted in On-line Table 1. Patients with VSS 3-4 were less likely to have an mRS 0-3, denoting ambulatory independence, in unadjusted analysis (OR, 0.43 ; 95\% CI, $0.25-0.73 ; P=.002)$ but not in the adjusted analysis (aOR, 0.75; 95\% CI, 0.40-1.43; $P=.38$ ). Ordered logistic regression revealed an overall shift toward a worse outcome in patients with VSS 3-4 with unadjusted common OR of 2.86 ; $95 \%$ CI, $1.83-4.52 ; P<.001$; and adjusted common OR of 3.04; 95\% CI, $1.93-4.84 ; P<.001$ (Fig 1). A shift toward worse outcomes with each incremental grade of VSS was also observed (Fig 1). Compared with VSS 0, patients with a baseline VSS 3 and 4 had higher odds of having a 90-day mRS 3-6 (OR, 3.58; 95\% CI, 1.618.64; $P=.003$; and OR 7.25; 95\% CI, 2.63-25.73; $P<.001$, 
respectively). After adjustment for covariables, VSS 4 continued to have a higher odds of 90-day mRS 3-6 (On-line Table 2).

Patients with VSS 3-4 demonstrated a higher likelihood of death by 90 days on unadjusted (OR, 1.97; 95\% CI, 1.06-3.57;

Table 1: Baseline characteristics of patients with no-to-minimal (van Sweeten score, 0-2) and moderate-to-severe (van Swieten score, 3-4) white matter disease

\begin{tabular}{|c|c|c|c|}
\hline & $\begin{array}{l}\text { van Swieten } \\
0-2(n=315)\end{array}$ & $\begin{array}{l}\text { van Swieten } \\
3-4(n=74)\end{array}$ & $P$ Value \\
\hline Age (mean) ( $\pm \mathrm{SD})(\mathrm{yr})$ & $66( \pm 15)$ & $76( \pm 12)$ & $<.001$ \\
\hline Male (\%) & $164(52)$ & $32(43)$ & .2 \\
\hline Hypertension (\%) & $224(71)$ & $68(92)$ & $<.001$ \\
\hline Diabetes (\%) & $92(29)$ & $23(31)$ & .78 \\
\hline Glucose (median) (IQR) & $122(102-146)$ & $131(115-162)$ & .009 \\
\hline NIHSS (median) (IQR) & $15(10-20)$ & 17 (11-21) & .16 \\
\hline ASPECTS (median) (IQR) & $8(7-10)$ & $8(7-9)$ & .07 \\
\hline Core volume (mean) ( $\pm S D$ ) & $20( \pm 24)$ & $19( \pm 26)$ & .85 \\
\hline Penumbra volume (mean) ( $\pm S D$ ) & $119( \pm 71)$ & $124( \pm 71)$ & .69 \\
\hline Systolic BP on admission (mean) ( \pm SD) & $154( \pm 29)$ & $168( \pm 29)$ & $<.001$ \\
\hline $\begin{array}{l}\text { Time from last known well to reperfusion } \\
\text { (median) (IQR) }\end{array}$ & $274(180-534)$ & $286(190-676)$ & .2 \\
\hline Intravenous thrombolysis (\%) & $160(51)$ & $29(39)$ & .09 \\
\hline Prior stroke (\%) & $52(16)$ & $19(26)$ & .09 \\
\hline
\end{tabular}

Note:-BP indicates blood pressure; IQR, interquartile range.
$P=.029$ ) but not on adjusted analysis (aOR, 1.14; 95\% CI, $0.56-2.26 ; P=.71$ ). The rates and odds of early neurologic improvement and successful recanalization were not significantly different among the 2 groups (Table 2). Notably, the rate of any $\mathrm{ICH}$ was also not significantly different between the 2 groups (22\%, $n=66$, in patients with VSS 02 versus $20 \%, n=15$, in patients with VSS $3-4 ; P=.86)$.

A successful recanalization was achieved in $343(88 \%)$ patients. Of these, 90-day outcome was available for 323 (94\%) patients. Outcomes of this subgroup of patients are outlined in Table 3. Overall, futile recanalization was observed in $80 \%$ of the patients with VSS 3-4 compared with $48 \%$ in those with VSS $0-2$ $(P<.001)$ when futile recanalization was defined as 90-day mRS 3-6 in patients with successful recanalization (OR, 4.19; 95\% CI, 2.24-8.37,
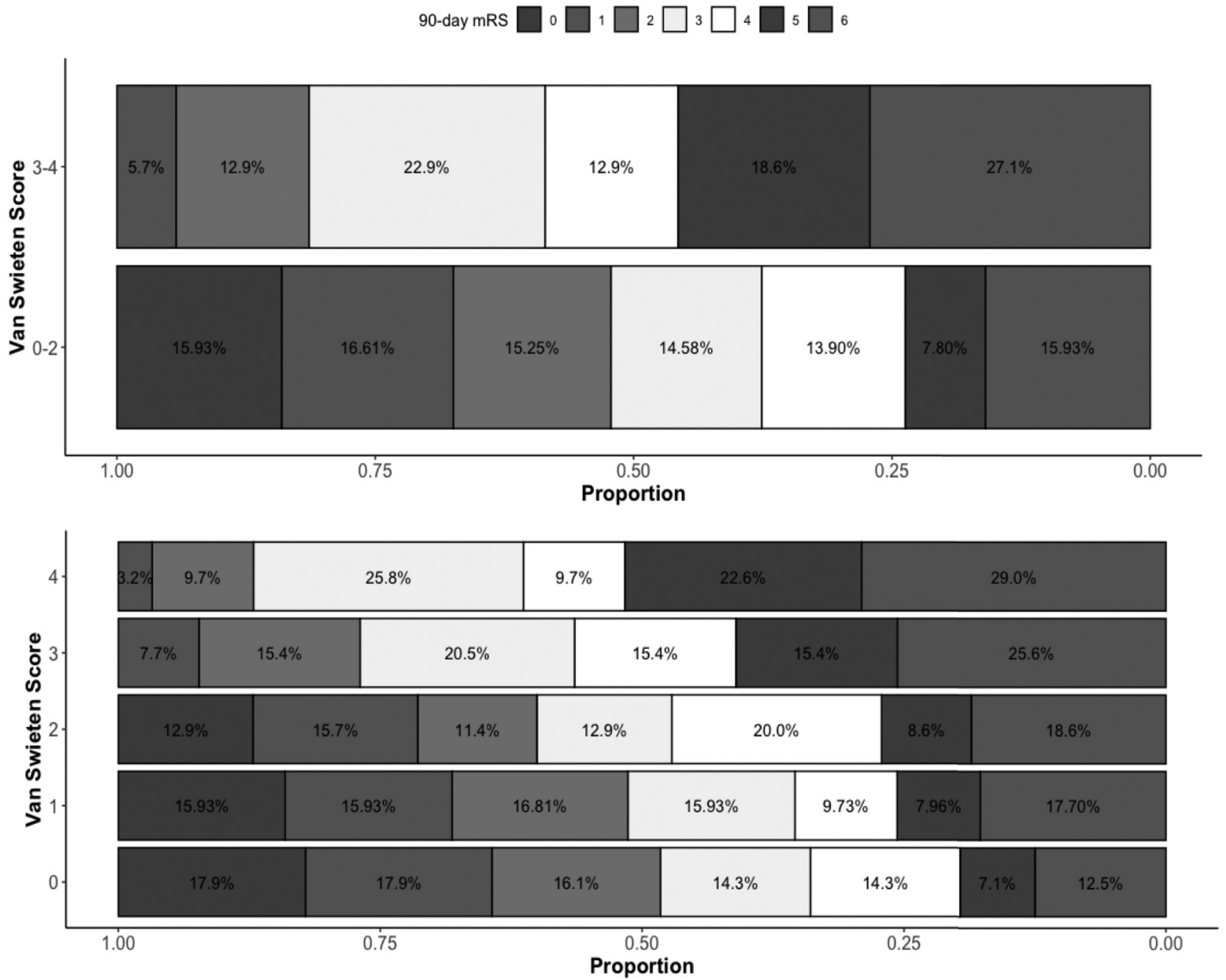

FIGURE. Distribution of the 90-day mRS score according to white matter disease severity graded with the van Swieten scale score. 
Table 2: Outcomes of patients with no-to-minimal (van Swieten score, 0-2) and moderate-to-severe (van Swieten score, 3-4) white matter disease

\begin{tabular}{|c|c|c|c|c|c|c|}
\hline Outcome & $\begin{array}{c}\text { van Swieten 0-2 } \\
(n=315)\end{array}$ & $\begin{array}{c}\text { van Swieten 3-4 } \\
(n=74)\end{array}$ & $\begin{array}{c}\text { Unadjusted OR } \\
\text { with } 95 \% \mathrm{Cl} \\
\end{array}$ & $P$ Value & $\begin{array}{l}\text { Adjusted OR } \\
\text { with } 95 \% \mathrm{Cl}\end{array}$ & $P$ Value \\
\hline 90-Day mRS 0-2 (\%) & $141 / 295(45)$ & $13 / 70(18)$ & $0.25(0.13-0.46)$ & $<.001$ & $0.37(0.17-0.75)$ & .008 \\
\hline 90-Day mRS 0-3 (\%) & $184 / 295(58)$ & $29 / 70(39)$ & $0.43(0.25-0.73)$ & .002 & $0.75(0.40-1.43)$ & .38 \\
\hline Shift in the 90 -day mRS & NA & NA & $2.86(1.83-4.52)$ & $<.001$ & $3.05(1.93-4.84)$ & $<.001$ \\
\hline Death $(\%)$ & $47(15)$ & $19(26)$ & $1.97(1.06-3.57)$ & .029 & $1.14(0.56-2.26)$ & .71 \\
\hline Symptomatic ICH (\%) & $13(4)$ & $4(5)$ & $1.33(0.37-3.88)$ & .629 & NA & NA \\
\hline $\mathrm{mTICl} \geq 2 \mathrm{~b}(\%)$ & 275 (87) & $68(92)$ & $1.65(0.72-4.47)$ & .27 & NA & NA \\
\hline ENI (\%) & $132(42)$ & $30(40)$ & $0.95(0.56-1.58)$ & .83 & NA & NA \\
\hline
\end{tabular}

Note:-mTICl indicates modified TICl; ENI, early neurologic improvement; NA, not applicable.

\begin{tabular}{|c|c|c|c|}
\hline Outcome & $\begin{array}{l}\text { van Swieten } \\
0-2(n=275)\end{array}$ & $\begin{array}{l}\text { van Swieten } \\
3-4(n=68)\end{array}$ & $P$ Value \\
\hline 90-Day mRS 0-2 (\%) & $132 / 258(52)$ & $13 / 65(20)$ & $<.001$ \\
\hline 90-Day mRS 0-3 (\%) & $169 / 258(65.5)$ & $28 / 65(43)$ & .002 \\
\hline Death (\%) & $34(12.4)$ & $17(25.0)$ & .013 \\
\hline Symptomatic ICH (\%) & $10(3.6)$ & $3(4.4)$ & .73 \\
\hline ENI (\%) & $124(45.1)$ & $30(44.1)$ & 1.0 \\
\hline
\end{tabular}

Note:-ENI indicates early neurologic improvement.

$P<.001 ;$ aOR, 2.59; 95\% CI, 1.26-5.65; $P=.012$ for 90 -day mRS 3-6). When defined as 90 -day mRS $4-6$, futile recanalization was observed in $57 \%$ of patients with VSS 3-4 versus $34.5 \%$ of those with VSS 0-2 (OR, 2.51; 95\% CI, 1.45-4.40; $P<.001 ;$ aOR, $1.34 ; 95 \% \mathrm{CI}, 0.7-2.57 ; P=.38$ for a 90 -day mRS 4-6). Rates of symptomatic ICH and death were similar in both groups. Among patients with VSS 3-4 who had a 90-day outcome available $(n=70)$, none of the patients with unsuccessful recanalization $(n=5)$ had a 90 -day mRS $0-2$ compared with 7/65 (20\%) patients with successful recanalization. Among those with VSS 0-2 and a 90-day outcome available $(n=295)$, 9/37 (24\%) patients with unsuccessful recanalization had a 90day mRS 0-2, compared with 132/258 (51\%) patients with successful recanalization (OR, 0.31 ; 95\% CI, 0.13-0.65; $P=.003$ ).

\section{DISCUSSION}

In this multicenter, prospective observational study, we found that patients with moderate-to-severe WMD had significantly worse functional outcomes at 90 days in terms of functional independence compared with those with no-to-minimal WMD (18\% versus $45 \% ; P<.001)$. A significant overall shift toward worse outcomes was also observed, though the rates of successful recanalization and hemorrhagic complications were not significantly different between the 2 groups. An increasing shift toward a worse outcome with each incremental grade of VSS may imply an underlying dose response between WMD and outcomes and suggests a possible causal relationship. Furthermore, the odds of futile recanalization were nearly 4 -fold higher in patients with moderate-to-severe WMD.

Acquired WMD is common with aging and has heterogeneous clinical and pathologic correlates. It most frequently represents the sequelae of chronic, inadequately controlled hypertension, which was an overwhelmingly common risk factor and probably the major driver of WMD in the current cohort. However, smoking, diabetes, cerebral amyloid angiopathy, rare hereditary cerebral small-vessel diseases, and even migraines have been reported to be linked to WMD. ${ }^{16,17}$ The presence of WMD on imaging is a useful biomarker for compromised cognitive performance, particularly on measures of memory and processing speed. ${ }^{17,18}$ Common pathologic correlates of WMD include those commonly implicated in neurodegeneration, such as elevated markers of axonal damage, the presence of activated microglia, and disorganization of aquaporin-4 localization on vessel-associated astrocytes. ${ }^{19-22}$

Mechanisms by which WMD may exert a negative effect on outcomes following an acute stroke are multifold. First, it is associated with disruption of endothelial function, resulting in damage to the microvascular integrity, and blood-brain barrier dysfunction, resulting in extravasation of blood products and hemorrhagic complications. ${ }^{23}$ Second, it may reduce the ischemic resilience of the cerebral tissue to a sudden lack of blood flow. ${ }^{24}$ Third, it can cause ongoing platelet activation and increased propensity for thrombosis, resulting in higher final infarct volumes. ${ }^{25}$ The resulting clinical consequences include not only worse outcomes with the incident stroke but also subsequent increased cognitive decline and rates of recurrent strokes. ${ }^{26,27}$

Outcomes of patients with moderate-to-severe WMD in acute reperfusion have mainly been studied in the context of intravenous thrombolysis. There is an increased rate of symptomatic $\mathrm{ICH}$ in patients with moderate-to-severe WMD. ${ }^{10,28}$ However, post hoc analysis of randomized data suggests that patients with moderate-to-severe WMD continue to derive the benefit from intravenous thrombolysis with an overall improvement in functional outcomes, compared with no thrombolytic treatment. ${ }^{28} \mathrm{~A}$ limited number of studies have reported the effect of WMD on outcomes after EVT. ${ }^{29-32}$ All of these studies were retrospective in design, most were single-center, likely included patients treated with older EVT devices, and included a relatively small number of patients.

The results of these studies have been variable. One study showed no difference in functional outcomes, ${ }^{29}$ and others have shown a worse functional outcome in moderate-to-severe WMD. $^{30,32}$ All the studies have consistently reported no difference in rates of hemorrhagic complications in contrast to similar studies involving intravenous thrombolysis. It is plausible that blood vessels rendered "leaky" from the microvascular damage from underlying WMD are more susceptible to the lytic effects of systemic thrombolysis. However, as the field of acute stroke 
advances, a heightened understanding of how varying substrates impact recovery will not only impact patient selection but may also lead to new targets for neurorestorative therapies.

Our study has the inherent limitations of an observational study. Most important, we did not include patients with baseline disability, likely introducing a selection bias. It is plausible that the outcomes may change after factoring the patients' disabled statuses, because patients with moderate-to-severe WMD are more likely to have pre-existing disability. Additionally, due to selection bias inherent to the inclusion criteria, our study population might not reflect patients with the most severe WMD and, therefore, may underestimate the negative impact of WMD on outcomes after EVT. Furthermore, without a placebo arm, it is impossible to know whether the effect of EVT is truly attenuated in patients with WMD. We may have been underpowered to detect a significant effect of WMD of hemorrhage rates. Our study is strengthened by a relatively large number of patients compared with prior studies, and its multi-institutional design makes the results more generalizable. Additionally, the patients included in this study are representative of the modern EVT population.

Our findings should be validated in large EVT cohorts, particularly those in randomized trials.

\section{CONCLUSIONS}

Moderate-to-severe WMD is associated with decreased rates of functional independence in patients undergoing EVT; however, there are no significant differences in the rates of procedural success or intracranial hemorrhage. Currently, our data cannot support withholding EVT on the basis of the severity of WMD but may provide some reassurance that the rate of symptomatic hemorrhage may not be significantly higher. Future analyses of randomized trial data are needed to understand whether the treatment effect of EVT is truly diminished in patients with moderate-to-severe WMD.

Disclosures: Eva A. Mistry-UNRELATED: Grants/Grants Pending: National Institutes of Health/National Institute of Neurological Disorders and Stroke, Vanderbilt Faculty Research Scholars Program, University of Cincinnati Gardner Neuroscience Institute, and Society of Vascular and Interventional Neurology, Comments: K23NS113858.* James E. Siegler-RELATED: Grant: National Institutes of Health, Comments: U10 NS086474 grant*; Support for Travel to Meetings for the Study or Other Purposes: American Heart Association, Comments: Junior Investigator Travel Award; UNRELATED: Payment for Lectures Including Service on Speakers Bureaus: Tufts Medical Center and Lahey Hospital \& Medical Center, Boston, Massachusetts, Comments: Grand Rounds honoraria. Scott E. Kasner-UNRELATED: Consultancy: Medtronic, Bayer, Bristol-Myers Squibb; Grants/Grants Pending: W. L. Gore \& Associates, Bayer, Bristol-Myers Squibb*; Royalties: Up-to-Date. Rohan ChitaleUNRELATED: Grants/Grants Pending: Cerenovus*; Payment for Lectures Including Service on Speakers Bureaus: Medtronic.* Michael Froehler-UNRELATED: Consultancy: Balt USA, Corindus Vascular Robotics, Genentech, Medtronic, Stryker, Viz.ai; Employment: Vanderbilt University Medical Center; Grants/Grants Pending: National Institutes of Health, Genentech, Stryker, Medtronic, MicroVention, Penumbra, Endophys.* Shadi Yaghi—UNRELATED: Consultancy: Medtronic.* Pooja Khatri-UNRELATED: Consultancy: Lumosa Therapeutics, Genentech, Comments: Data and Safety Monitoring Board (Lumosa), trial Principal Investigator (Genentech)*; Expert Testimony: medicolegal consultation*; Grants/Grants Pending: Cerenovus, Nervive.* *Money paid to the institution.

\section{REFERENCES}

1. Powers WJ, Rabinstein AA, Ackerson T, et al; American Heart Association Stroke Council. 2018 guidelines for the early management of patients with acute ischemic stroke: a guideline for healthcare professionals from the American Heart Association/ American Stroke Association. Stroke 2018;49:e46-110 CrossRef Medline

2. Goyal M, Menon BK, van Zwam WH, et al; HERMES Collaborators. Endovascular thrombectomy after large-vessel ischaemic stroke: a meta-analysis of individual patient data from five randomised trials. Lancet 2016;387:1723-31 CrossRef Medline

3. Smith EE, Saver JL, Cox M, et al. Increase in endovascular therapy in Get with the Guidelines-Stroke after the Publication of Pivotal Trials. Circulation 2017;136:2303-10 CrossRef Medline

4. Albers GW, Marks MP, Kemp S, et al; DEFUSE 3 Investigators. Thrombectomy for stroke at $\mathbf{6}$ to 16 hours with selection by perfusion imaging. N Engl J Med 2018;378:708-18 CrossRef Medline

5. Nogueira RG, Jadhav AP, Haussen DC, et al; DAWN Trial Investigators. Thrombectomy 6 to 24 hours after stroke with a mismatch between deficit and infarct. N Engl J Med 2018;378:11-21 CrossRef Medline

6. Roman LS, Menon BK, Blasco J, et al; HERMES Collaborators. Imaging features and safety and efficacy of endovascular stroke treatment: a meta-analysis of individual patient-level data. Lancet Neurol 2018;17:895-904 CrossRef Medline

7. The TELSA trial. Thrombectomy for Emergent Salvage of Large Anterior Circulation Ischemic Stroke. https:/www.cms.gov/Medicare/Coverage/ IDE/Approved-IDE-Studies-Items/G190006-NCT03805308. Accessed January 31, 2020

8. Sarraj A, Hassan AE, Savitz S, et al. Outcomes of endovascular thrombectomy vs medical management alone in patients with large ischemic cores: a secondary analysis of the Optimizing Patient's Selection for Endovascular Treatment in Acute Ischemic Stroke (select) study. JAMA Neurol 2019; Jul 29. [Epub ahead of print] CrossRef Medline

9. Nagel S, Bouslama M, Krause LU, et al. Mechanical thrombectomy in patients with milder strokes and large vessel occlusions. Stroke 2018;49:2391-97 CrossRef Medline

10. Kongbunkiat K, Wilson D, Kasemsap N, et al. Leukoaraiosis, intracerebral hemorrhage, and functional outcome after acute stroke thrombolysis. Neurology 2017;88:638-45 CrossRef Medline

11. Smith EE. Leukoaraiosis and stroke. Stroke 2010;41:S139-43 CrossRef Medline

12. Mistry EA, Sucharew H, Mistry AM, et al. Blood Pressure after Endovascular Therapy for Ischemic Stroke (BEST): a multicenter prospective cohort study. Stroke 2019;50:3449-55 CrossRef Medline

13. van Swieten JC, Hijdra A, Koudstaal PJ, et al. Grading white matter lesions on CT and MRI: a simple scale. J Neurol Neurosurg Psychiatry 1990;53:1080-83 CrossRef Medline

14. Boulouis G, Bricout N, Benhassen W, et al. White matter hyperintensity burden in patients with ischemic stroke treated with thrombectomy. Neurology 2019;93:e1498-1506 CrossRef Medline

15. Pantoni L, Simoni M, Pracucci G, et al. Visual rating scales for agerelated white matter changes (leukoaraiosis): can the heterogeneity be reduced? Stroke 2002;33:2827-33 CrossRef Medline

16. Erdelyi-Botor S, Aradi M, Kamson DO, et al. Changes of migrainerelated white matter hyperintensities after 3 years: a longitudinal MRI study. Headache 2015;55:55-70 CrossRef Medline

17. Habes M, Erus G, Toledo JB, et al. White matter hyperintensities and imaging patterns of brain ageing in the general population. Brain 2016;139:1164-79 CrossRef Medline

18. Schrag M, Kirshner H. Neuropsychological effects of cerebral amyloid angiopathy. Curr Neurol Neurosci Rep 2016;16:76 CrossRef Medline

19. Chen A, Akinyemi RO, Hase Y, et al. Frontal white matter hyperintensities, clasmatodendrosis and gliovascular abnormalities in ageing and post-stroke dementia. Brain 2016;139:242-58 CrossRef Medline

20. Moore EE, Hohman TJ, Badami FS, et al. Neurofilament relates to white matter microstructure in older adults. Neurobiol Aging 2018;70:233-41 CrossRef Medline

21. Osborn KE, Alverio JM, Dumitrescu L, et al. Adverse vascular risk relates to cerebrospinal fluid biomarker evidence of axonal injury 
in the presence of Alzheimer's disease pathology. J Alzheimers Dis 2019;71:281-90 CrossRef Medline

22. Raz N, Yang Y, Dahle CL, et al. Volume of white matter hyperintensities in healthy adults: contribution of age, vascular risk factors, and inflammation-related genetic variants. Biochim Biophys Acta 2012;1822:361-69 CrossRef Medline

23. del Zoppo GJ, von Kummer R, Hamann GF. Ischaemic damage of brain microvessels: inherent risks for thrombolytic treatment in stroke. J Neurol Neurosurg Psychiatry 1998;65:1-9 CrossRef Medline

24. Henninger N, Khan MA, Zhang J, et al. Leukoaraiosis predicts cortical infarct volume after distal middle cerebral artery occlusion. Stroke 2014;45:689-95 CrossRef Medline

25. Iwamoto T, Kubo H, Takasaki M. Platelet activation in the cerebral circulation in different subtypes of ischemic stroke and Binswanger's disease. Stroke 1995;26:52-56 CrossRef Medline

26. Inzitari D, Pracucci G, Poggesi A, et al; LADIS Study Group. Changes in white matter as determinant of global functional decline in older independent outpatients: three-year follow-up of LADIS (Leukoaraiosis and Disability] study cohort. BMJ 2009;339: b2477 CrossRef Medline
27. Vermeer SE, Hollander M, van Dijk EJ, et al, Rotterdam Scan Study. Silent brain infarcts and white matter lesions increase stroke risk in the general population: the Rotterdam Scan study. Stroke 2003;34:1126-29 CrossRef Medline

28. Demchuk AM, Khan F, Hill MD, et al; NINDS rt-PA Stroke Study Group. Importance of leukoaraiosis on CT for tissue plasminogen activator decision making: evaluation of the NINDS rt-PA Stroke study. Cerebrovasc Dis 2008;26:120-25 CrossRef Medline

29. Atchaneeyasakul K, Leslie-Mazwi T, Donahue K, et al. White matter hyperintensity volume and outcome of mechanical thrombectomy with Stentriever in acute ischemic stroke. Stroke 2017;48:2892-94 CrossRef Medline

30. Guo Y, Zi W, Wan Y, et al. Leukoaraiosis severity and outcomes after mechanical thrombectomy with stent-retriever devices in acute ischemic stroke. J Neurointerv Surg 2019;11:137-40 CrossRef Medline

31. Shi ZS, Loh Y, Liebeskind DS, et al. Leukoaraiosis predicts parenchymal hematoma after mechanical thrombectomy in acute ischemic stroke. Stroke 2012;43:1806-11 CrossRef Medline

32. Zhang J, Puri AS, Khan MA, et al. Leukoaraiosis predicts a poor 90-day outcome after endovascular stroke therapy. AJNR Am J Neuroradiol 2014;35:2070-75 CrossRef Medline 